\title{
385. Behelfsorthesen - Indikation und Technik
}

\author{
G. Aldinger
}

Orthopädische Klinik und Poliklinik der Universität Tübingen (Direktor: Prof. Dr. H. Mau), Calwer Straße 7, D-7400 Tübingen

\section{Temporary Orthoses - Indication and Technique}

Summary. A temporary orthosis can be easily, quickly and cheaply manufactured by splitting and cutting a polyurethane plaster. The orthosis is used for stabilization of bones and joints and can be removed for function, physiotherapy, inspection of wounds and personal hygiene. This method of bracing allows individual treatment, even of short duration.

Key words: Plaster - Orthosis.

Zusammenfassung. Eine Behelfsorthese läßt sich leicht, schnell und kostengünstig durch einfaches Spalten und Zurechtschneiden eines Polyurethan-Kunststoff-Stützverbandes überall und ohne spezielle Kenntnisse herstellen. Sie wird zur Stabilisierung von Knochen und Gelenken angelegt und kann zur Krankengymnastik und Funktion sowie zur Wundkontrolle, Haut- und Körperpflege wieder abgenommen werden. Auch bei kurzer Behandlungsdauer wird hierdurch eine orthetische Versorgung möglich. Die Behelfsorthese bietet dem kreativen Therapeuten eine Fülle von Möglichkeiten und schafft die Voraussetzungen für eine situationsgerechte, den pathomorphologischen Gegebenheiten angepaßte Therapie.

Schlïsselwörter: Stützverband -- Orthese.

\section{Operative Therapie des Pankreascarcinoms}

\author{
Ch. Gebhardt, F. P. Gall, P. Hermanek, H. Zirngibl und A. Altendorf \\ Chirurgische Klinik und Abteilung für klinische Pathologie der Universität Erlangen-Nürnberg, \\ Krankenhausstraße 12, D-8520 Erlangen
}

\section{Operative Treatment of Pancreatic Cancer}

Summary. Report on 417 operations for periampullary and ductal pancreatic cancer. Between 1979 and 1981 the resection rates were increased to $85 \%$ and $29 \%$, respectively. Prognosis for periampullary carcinoma was better than for ductal pancreatic cancer because the diagnosis was established at an earlier tumor stage. However, in cases with pNO (without lymph node metastases) we found a median survival time of more than 60 months for the periampullary as well as the ductal carcinoma cases.

Key words: Pancreatic carcinoma - Survival times.

Zusammenfassung. Bericht über 417 Eingriffe wegen periampullärer und duktaler Pankreascarcinome. Die Resektionsquoten konnten in den Jahren 1979-1981 auf $85 \%$ bzw. $29 \%$ angehoben werden. Die Prognose des periampullären Carcinoms war nur günstiger als die des duktalen Carcinoms, weil die Diagnose in einem früheren Tumorstadium gestellt wurde. Bei pNO, also fehlenden Lymphknotenmetastasen, lag die mediane Überlebenszeit beim periampullären und duktalen Carcinom über 60 Monate.

Schlïsselwörter: Pankreascarcinom - Überlebenszeiten. 